\title{
Numerical Integration of Functions with Endpoint Singularities and/or Complex Poles in 3D Galerkin Boundary Element Methods*
}

\author{
To Professor Masatake Mori for his outstanding contribution \\ to numerical integration
}

By

Giovanni Monegato and Letizia Scuderi**

\begin{abstract}
In this paper we propose special strategies to compute $1 \mathrm{D}$ integrals of functions having weakly or strong singularities at the endpoints of the interval of integration or complex poles close to the domain of integration. As application of the proposed strategies, we compute a four dimensional integral arising from 3D Galerkin boundary element methods (BEM) applied to hypersingular boundary integral equations.
\end{abstract}

\section{§1. Introduction}

In the computation of integrals and in the numerical solution of integral equations, one often has to deal with the numerical integration of functions with endpoint weak singularities, or with analytic functions having complex conjugate poles near the domain of integration. Nonlinear changes of variables have shown to be a very effective tool to face these problems. Indeed, in the case of weak singularities a proper change of variable can make the integrand function arbitrarily smooth. This, combined with the use of a standard rule

Communicated by H. Okamoto. Received September 28, 2004. Revised February 22, 2005.

2000 Mathematics Subject Classification(s): 65D32, 65R20, 65N38.

*This work was supported by the Ministero dell'Istruzione, dell'Università e della Ricerca of Italy and by the INDAM 2003 project "Modellistica Numerica per il Calcolo Scientifico e Applicazioni Avanzate".

** Dipartimento di Matematica, Politecnico di Torino, Corso Duca degli Abruzzi 24, 10129 Torino, Italy. 
like the Gauss-Legendre one or the composite trapezoidal formula, allows to achieve high accuracy using a low number of abscissas (see [2], [8], [9], [11], [14], [16]). Here we recall some of the known changes of variable, all having as co-domain the interval $(0,1)$, which have been proposed in the literature. For some comparisons (see [9]).

In [8], the following polynomial transformation, which is a generalization of the one proposed in [6] to construct lattice rules for multiple integration on the hypercube, has been used:

$$
\varphi_{1}(t)=\frac{(p+q-1)}{(p-1)(q-1)} \int_{0}^{t} u^{p-1}(1-u)^{q-1} d u, 0 \leq t \leq 1, p, q \geq 1 .
$$

The integral in (1.1) could be computed, for any $t \in(0,1]$, using recurrence relations (see [9]). In any case it can be evaluated more efficiently, for the given values of $p$ and $q$, by means of the $n$-point Gauss-Legendre rule, with $n=\left\lfloor\frac{p+q}{2}\right\rfloor$.

Then, we recall a trigonometric transformation of the form

$$
\varphi_{2}(t)=\frac{\int_{0}^{t}\left(\sin \frac{\pi}{2} u\right)^{p-1}\left(\cos \frac{\pi}{2} u\right)^{q-1} d u}{\int_{0}^{1}\left(\sin \frac{\pi}{2} u\right)^{p-1}\left(\cos \frac{\pi}{2} u\right)^{q-1} d u}, 0 \leq t \leq 1, p, q \geq 1 .
$$

This latter generalizes a trigonometric transformation proposed in [14]. Also in this case the integral in (1.2) could be computed for any $t \in(0,1]$ by using proper recurrence relations or by a direct computation using a Gauss-Legendre rule.

A third transformation, of rational type, used in [11], [3] in connection with the solution of certain integral equations, and in [4] to evaluate Hadamard finite-part integrals, is the following one:

$$
\varphi_{3}(t)=\frac{t^{p}}{t^{p}+(1-t)^{q}}, 0 \leq t \leq 1, p, q \geq 1 .
$$

The last transformation we recall is of exponential type:

$$
\varphi_{4}(t)=\frac{1}{2}+\frac{1}{2} \tanh \left(\frac{\pi}{2} \sinh (t)\right),-\infty<t<\infty .
$$

It is the well-known double exponential (DE) transformation (see [16], [10], $[15])$.

In this paper we examine the use of nonlinear changes of variable for the numerical evaluation of some 4-dimensional integrals arising in the numerical solution of hypersingular boundary integral equations. In particular we consider 
3D Galerkin boundary element methods, where a key issue is the efficient and fast computation of integrals of the form

$$
\int_{\Delta_{i}} \int_{\Delta_{j}} \frac{k(x, y)}{\|x-y\|^{3}} d y d x,
$$

where $\Delta_{i}, \Delta_{j}$ are triangular elements, possibly curved, and $k(x, y)$ is bounded.

When $\Delta_{i}$ and $\Delta_{j}$ are disjoint and not too close to each other, the computation of the above integral can be performed by standard rules. The difficult cases are those where (i) $\Delta_{i}$ and $\Delta_{j}$ are coincident, or share a common edge, or (ii) are disjoint but very close to each other. When $\Delta_{i}$ and $\Delta_{j}$ have only a common point, but are not close to each other at some other points, the computation can be classified standard.

Many papers have been devoted to this topic (see, for example, [1], [5], [12], [13]), although none of them seems to treat the case (ii), which is considered by some users the most "difficult" case. However in our opinion none of them are fully satisfactory because generally they either require some analytical calculation which is by non means trivial, or do not take into account the presence of complex poles which may effect adversely the numerical computation.

Indeed the strategy adopted by these methods is that of reducing the computation of the multidimensional integral to the evaluation of nested 1D integrals of analytic functions. However this is still not satisfactory because it is by now well-known that when integrating an analytic function, the position of its poles is crucial for the performance of a quadrature rule. When an analytic function has poles which are outside the domain of integration but lie very close to it, and this is what happens in the computation of some of our integrals, its numerical integration by means of a quadrature rule can give very poor accuracy if one does not adopt a special strategy.

In this paper we examine the case of (1.5) with $\Delta_{i}, \Delta_{j}$ coinciding. The other cases of $\Delta_{i}, \Delta_{j}$ having a common edge or disjoint but very close to each other will be treated in a following paper. We believe that our strategy can be applied successfully also to these cases.

\section{$\S 2$. One-dimensional Integration Rules}

There are two typical approaches to compute (1.5). One uses the global cartesian coordinates (see [5]), the other uses local polar coordinates (see [12]). Both of them reduce (1.5) to four nested one-dimensional integrals, whose integrand functions are analytical in the first case, and analytical or with logsingularities at the endpoints of the interval of integration in the second case. 
Unfortunately, in both cases the generated analytical functions have complex poles, depending upon outer variables or the form of the triangles, which may be arbitrarily close to the interval of integration. Therefore, for both approaches the Gaussian quadrature rules perform very poorly unless one considers a high number of quadrature nodes. Recalling that we have to compute four nested one-dimensional integrals, we can use only a few (let us say, at the most $6 \div 10$ ) quadrature nodes for each integral. Notice that 10 quadrature nodes for each integral means a total of $10^{4}$ quadrature nodes to compute (1.5), which represents only one element of the system matrix arising from the Galerkin boundary element method. For this reason in this section we propose special strategies, which allow us to compute the integrals with the above-mentioned characteristics using very few Gaussian quadrature nodes. In particular we propose to use variable transformations of the type (1.1), either to make the integrand functions smoother at the endpoints of the interval of integration, or to move poles away from the interval. Then, the Gauss-Legendre rule is applied.

In these situations the DE-rule, which is obtained by combining (1.4) with the (truncated) trapezoidal rule (see [16]), has certainly a much higher (exponential) rate of convergence. However, in the particular application we will consider in Section 3, it is of key importance to obtain a good accuracy with a very small number of function evaluations. For such small values of $n$, let us say $n \leq 10$, our approach appears more efficient.

A typical case is an integral of a function having only a log-singularities at the endpoints of the interval of integration. For example,

$$
I_{1}=\int_{0}^{1} e^{x} \log \frac{1-x}{x} d x .
$$

By introducing in (2.1) the change of variable (1.1), that here we write in the form

$$
x=\gamma_{0,1}^{q_{0}, q_{1}}(t):=\frac{\left(q_{0}+q_{1}-1\right)}{\left(q_{0}-1\right)\left(q_{1}-1\right)} \int_{0}^{t} x^{q_{0}-1}(1-x)^{q_{1}-1} d x, q_{0}, q_{1} \geq 1,
$$

and then applying the $n$-point Gauss-Legendre rule we obtain the improvements reported in Table 1 . Notice that in (2.2) for $q_{1}=1$ we have

$$
\gamma_{0,1}^{q_{0}, 1}(t)=t^{q_{0}}=: \gamma_{0}^{q_{0}}(t)
$$

and for $q_{0}=1$

$$
\gamma_{0,1}^{1, q_{1}}(t)=1-(1-t)^{q_{1}}=: \gamma_{1}^{q_{1}}(t)
$$


the choices (2.3) and (2.4) allow to smooth the singularities at the endpoints 0 and 1 , respectively. If $q_{0}=q_{1}=q$, we set

$$
\gamma_{0,1}^{q}:=\gamma_{0,1}^{q, q}
$$

\begin{tabular}{|c||c|c|c|c|}
\hline$n$ & $q=1$ & $q=2$ & $q=3$ & $q=4$ \\
\hline 2 & $2.50-01$ & $5.65-01$ & $9.07-01$ & $9.04-01$ \\
4 & $6.67-02$ & $1.35-02$ & $9.56-03$ & $1.34-01$ \\
8 & $1.80-02$ & $1.21-03$ & $1.87-04$ & $6.83-05$ \\
16 & $4.72-03$ & $8.32-05$ & $3.09-06$ & $2.00-07$ \\
32 & $1.21-03$ & $5.49-06$ & $5.17-08$ & $8.34-10$ \\
64 & $3.08-04$ & $3.53-07$ & $8.40-10$ & $3.36-12$ \\
128 & $7.75-05$ & $2.24-08$ & $1.31-11$ & $2.27-13$ \\
256 & $1.95-05$ & $1.41-09$ & $7.81-14$ & $1.38-13$ \\
512 & $4.87-06$ & $8.89-11$ & -- & -- \\
\hline
\end{tabular}

Table 1. Relative errors in $I_{1}=\int_{0}^{1} e^{\gamma(t)} \log \frac{1-\gamma(t)}{\gamma(t)} \gamma^{\prime}(t) d t, \gamma(t)=\gamma_{0,1}^{q}(t)$.

In all tables the sign "-_" means that full relative accuracy (i.e. 14 significant digits in our case) has been achieved.

Another situation that we will meet is that of integrals of the form

$$
I_{2}=\int_{0}^{1} \frac{f(x)}{x^{2}+\varepsilon^{2}} d x
$$

with $\varepsilon$ small and $f(x)$ smooth. To compute efficiently this integral it is convenient to introduce preliminarily the simple change of variable

$$
x=\gamma_{0}^{q}(t), q>1,
$$

and then to apply the $n$-point Gauss-Legendre rule. For example, if we take $f(x)=e^{x}$ we obtain the relative errors of Table 2 .

\begin{tabular}{|c||c|c||c|c||c|c|}
\hline \multicolumn{1}{|c||}{} & \multicolumn{2}{c||}{$\varepsilon=10^{-1}$} & \multicolumn{2}{c||}{$\varepsilon=10^{-3}$} & \multicolumn{2}{c|}{$\varepsilon=10^{-5}$} \\
\hline$n$ & $q=1$ & $q=2$ & $q=1$ & $q=4$ & $q=1$ & $q=7$ \\
\hline 4 & $4.25-02$ & $6.11-03$ & $9.72-01$ & $6.31-01$ & $1.00+00$ & $8.88-01$ \\
8 & $1.45-03$ & $5.75-04$ & $9.05-01$ & $1.71-01$ & $9.99-01$ & $2.74-01$ \\
16 & $9.75-07$ & $1.20-07$ & $6.61-01$ & $2.73-03$ & $9.96-01$ & $1.11-01$ \\
32 & $1.98-13$ & -- & $7.20-02$ & $3.16-05$ & $9.87-01$ & $2.85-03$ \\
64 & -- & -- & $6.30-03$ & $1.29-10$ & $9.47-01$ & $4.50-07$ \\
128 & -- & -- & $3.53-05$ & -- & $7.92-01$ & $1.43-12$ \\
256 & -- & -- & $3.44-10$ & -- & $2.90-01$ & -- \\
512 & -- & -- & -- & -- & $3.85-02$ & -- \\
\hline
\end{tabular}

Table 2. Relative errors in $I_{2}=\int_{0}^{1} \frac{q t^{q-1} t^{t^{q}}}{t^{2 q}+\varepsilon^{2}} d t$.

Since our 1D rules will be applied to a quadruple integral, it is important to obtain the required accuracy using a small number of nodes. In the integral 
(2.6), when $\varepsilon$ is very small, for example in the above case $\varepsilon=10^{-5}$, the value of $q$ one has to take makes the new integrand function so flat around the origin, that to achieve a good accuracy one has to take a value of $n$ which is not any longer small. In this situation it is more convenient to proceed as follows. Write

$$
I_{2}=\left(\int_{0}^{\varepsilon}+\int_{\varepsilon}^{1}\right) \frac{f(x)}{x^{2}+\varepsilon^{2}} d x
$$

then apply the $n$-point Gauss-Legendre rule to the first integral on the righthand side, and to the second one after having introduced in it the change of variable $x=\gamma_{0}^{q}(t)$. Subdivision (2.7) is of key importance for the following reasons. In (2.6) the transformation $x=\gamma_{0}^{q}(t)$ leaves unchanged the interval of integration, but as $q$ gets larger, the modulus of the poles tends to 1, and after a certain value of $q$ some of the poles move again towards the interval of integration. In (2.7) this unwanted phenomenon does not arise. Indeed, in the first integral on the right-hand side the distance of the two poles from the interval of integration is equal to the length of this latter, while the length of the interval of integration of the second integral, after having introduced the transformation $x=\gamma_{0}^{q}(t)$, tends to zero as $q$ tends to infinity. The effectiveness of this new approach is shown by the results in Table 3 .

\begin{tabular}{|c||c|c||c|c||c|c|}
\hline \multicolumn{1}{|c||}{} & \multicolumn{2}{c||}{$\varepsilon=10^{-1}$} & \multicolumn{2}{c||}{$\varepsilon=10^{-3}$} & \multicolumn{2}{c|}{$\varepsilon=10^{-5}$} \\
\hline $2 n$ & $q=3$ & $q=100$ & $q=4$ & $q=100$ & $q=7$ & $q=100$ \\
\hline 4 & $8.26-03$ & $6.04-03$ & $3.00-01$ & $1.28-02$ & $4.40-01$ & $2.04-01$ \\
8 & $6.07-05$ & $1.15-05$ & $1.31-03$ & $4.50-03$ & $8.36-02$ & $3.42-02$ \\
16 & $5.44-09$ & $2.11-09$ & $2.93-04$ & $3.20-05$ & $9.46-03$ & $4.90-04$ \\
32 & -- & -- & $7.74-07$ & $5.01-10$ & $2.38-05$ & $7.69-08$ \\
64 & -- & -- & $6.54-13$ & -- & $5.05-10$ & -- \\
\hline
\end{tabular}

Table 3. Relative errors in $I_{2}=\int_{0}^{\varepsilon} \frac{e^{x}}{x^{2}+\varepsilon^{2}} d x+\int_{\varepsilon^{q}}^{1} \frac{q t^{q-1} e^{t^{q}}}{t^{2 q}+\varepsilon^{2}} d t$.

Similarly, if the integral has the form

$$
I_{3}=\int_{a}^{b} \frac{f(x)}{\left(x^{2}+\varepsilon^{2}\right)^{\alpha}} d x
$$

with $a<0, b>0$ and $\alpha>0$, then we proceed as in (2.7) by splitting (2.8) as follows

$$
I_{3}=\left(\int_{a}^{-\varepsilon}+\int_{-\varepsilon}^{\varepsilon}+\int_{\varepsilon}^{b}\right) \frac{f(x)}{\left(x^{2}+\varepsilon^{2}\right)^{\alpha}} d x .
$$

We apply the $n$-point Gauss-Legendre quadrature rule to each integral, after having introduced the change of variable $x=t^{q}$ in the first and in the third 
integral to the right-hand side of (2.9). In the following Table 4, we have reported the relative errors obtained by this last numerical procedure when in (2.8) $f \equiv 1, a=-0.5, b=0.5$ and $\alpha=3 / 2$.

In Table 4 and below $G L_{m}$ denotes the $m$-point Gauss-Legendre rule.

\begin{tabular}{|c||c|c||c|c|c||c|c|c|}
\hline \multicolumn{1}{|c||}{} & \multicolumn{2}{c||}{$\varepsilon=10^{-1}$} & \multicolumn{3}{c||}{$\varepsilon=10^{-3}$} & \multicolumn{3}{c|}{$\varepsilon=10^{-5}$} \\
\hline $3 n$ & $G L_{3 n}$ & $q=2$ & $G L_{3 n}$ & $q=4$ & $q=100$ & $G L_{3 n}$ & $q=7$ & $q=100$ \\
\hline 6 & $3.15-01$ & $5.98-02$ & $1.00+00$ & $3.11-01$ & $1.56-01$ & $1.00+00$ & $3.48-01$ & $3.04-01$ \\
12 & $4.17-02$ & $2.31-03$ & $9.99-01$ & $2.96-02$ & $8.03-03$ & $1.00+00$ & $1.57-01$ & $1.36-02$ \\
18 & $4.62-03$ & $8.27-05$ & $9.99-01$ & $9.16-03$ & $7.72-04$ & $1.00+00$ & $1.87-03$ & $4.98-03$ \\
24 & $4.85-04$ & $2.77-06$ & $9.98-01$ & $6.75-04$ & $3.02-05$ & $1.00+00$ & $1.30-02$ & $9.44-04$ \\
30 & $4.96-05$ & $9.06-08$ & $9.97-01$ & $2.20-04$ & $9.70-07$ & $1.00+00$ & $1.74-03$ & $1.95-05$ \\
36 & $4.97-06$ & $2.91-09$ & $9.95-01$ & $1.34-05$ & $3.02-08$ & $1.00+00$ & $6.08-04$ & $1.37-05$ \\
72 & $4.24-12$ & -- & $9.82-01$ & $6.12-11$ & -- & $1.00+00$ & $1.38-07$ & $5.82-11$ \\
144 & -- & -- & $9.32-01$ & -- & -- & $1.00+00$ & -- & -- \\
\hline
\end{tabular}

Table 4. Relative errors in $I_{3}=\left(\int_{\varepsilon^{\frac{1}{q}}}^{|a|^{\frac{1}{q}}}+\int_{\varepsilon^{\frac{1}{q}}}^{b^{\frac{1}{q}}}\right) q t^{q-1}\left(t^{2 q}+\varepsilon^{2}\right)^{-3 / 2} d t$

$$
+\int_{-\varepsilon}^{\varepsilon}\left(x^{2}+\varepsilon^{2}\right)^{-3 / 2} d x, a=-0.5, b=0.5 \text {. }
$$

The integral we have to compute could also be of the type

$$
I_{4}=\int_{0}^{1} \frac{f(x)}{\left[(x-r)^{2}+\varepsilon^{2}\right]^{\alpha}} d x
$$

with $0<r<1$ and $\alpha>0$. Notice that also $I_{3}$ can be rewritten in this form and vice versa. In this case we can proceed as follows. Write first

$$
I_{4}=\left(\int_{0}^{r}+\int_{r}^{1}\right) \frac{f(x)}{\left[(x-r)^{2}+\varepsilon^{2}\right]^{\alpha}} d x,
$$

and then

$$
I_{4}=\frac{1-r}{r} \int_{r}^{1} \frac{f\left(\frac{r}{1-r}(1-t)\right)}{\left[(t-r)^{2}+\left(\frac{1-r}{r} \varepsilon\right)^{2}\right]^{\alpha}} d t+\int_{r}^{1} \frac{f(x)}{\left[(x-r)^{2}+\varepsilon^{2}\right]^{\alpha}} d x .
$$

This splitting is suggested by the remark that, when one uses Gaussian rules, poles with real parts close to the endpoints of the interval of integration are less adverse than poles with a real part in the central part of the same interval. Finally, we introduce, as for $I_{2}$, the change of variable $t, x=u^{q}, q \geq 1$, in both integrals and apply the same Gauss-Legendre rule to each of them.

Since this strategy is also alternative to a splitting of the type (2.9), we have applied it to the same integral considered in Table 4 . Therefore, in Table 5 we have reported the relative errors obtained by taking in $(2.10) f \equiv 1, r=0.5$ and $\alpha=3 / 2$. 


\begin{tabular}{|c||c|c|c||c|c|c|}
\hline \multicolumn{1}{|c||}{} & \multicolumn{3}{c||}{$\varepsilon=10^{-1}$} & \multicolumn{3}{c|}{$\varepsilon=5 \cdot 10^{-2}$} \\
\hline $2 n$ & $G L_{2 n}$ & $q=1$ & $q=50$ & $G L_{2 n}$ & $q=1$ & $q=50$ \\
\hline 6 & $3.15-01$ & $6.77-01$ & $4.95-02$ & $7.14-01$ & $1.46-01$ & $3.80-02$ \\
12 & $4.17-02$ & $2.49-03$ & $2.55-06$ & $3.34-01$ & $2.25-02$ & $3.06-03$ \\
18 & $4.62-03$ & $6.05-05$ & $9.70-06$ & $1.27-01$ & $2.19-03$ & $1.83-04$ \\
24 & $4.85-04$ & $1.03-06$ & $4.48-08$ & $4.43-02$ & $1.54-04$ & $1.00-05$ \\
30 & $4.96-05$ & $9.08-09$ & $1.28-09$ & $1.48-02$ & $7.72-06$ & $5.18-07$ \\
36 & $4.97-06$ & $1.60-10$ & $1.21-11$ & $4.86-03$ & $1.62-07$ & $2.58-08$ \\
72 & $4.24-12$ & -- & -- & $5.07-06$ & -- & -- \\
144 & -- & -- & -- & $4.04-12$ & -- & -- \\
\hline
\end{tabular}

Table 5. Relative errors in $I_{4}=\frac{1-r}{r} \int_{r^{q}}^{1} q u^{q-1} f\left(\frac{r}{1-r}\left(1-u^{q}\right)\right)\left[\left(u^{q}-r\right)^{2}\right.$ $\left.+\left(\frac{1-r}{r} \varepsilon\right)^{2}\right]^{-3 / 2} d u+\int_{r^{q}}^{1} q u^{q-1} f\left(u^{q}\right)\left[\left(u^{q}-r\right)^{2}+\varepsilon^{2}\right]^{-3 / 2} d u, r=0.5$.

From a comparison between the Tables 4 and 5, it appears that (2.11) is more effective when $\varepsilon$ is not too small; let us say of order greater than $10^{-2}$. Otherwise (2.9) seems to produce better results.

The above strategy has been applied also to similar integrals with the algebraic polynomial (of degree 2) replaced by a corresponding trigonometric polynomial. In particular, we have also considered integrals of the type

$$
I_{5}=\int_{a}^{b} \frac{f(x)}{\left[\left(a_{1} \cos (x)+b_{1} \sin (x)\right)^{2}+\left(a_{2} \cos (x)+b_{2} \sin (x)\right)^{2}\right]^{\alpha}} d x .
$$

By $z_{0}$ we denote the pair of complex conjugate poles of the trigonometric polynomial closest to the interval of integration. In this situation, we have applied the above strategy by choosing $r=\operatorname{Re}\left(z_{0}\right)$. The results we have obtained by taking in (2.12) $f \equiv 1, a=-1.5, b=0, \alpha=3 / 2$ and $a_{1}=0, a_{2}=b_{2}=1$, $b_{1}=c$ with varying $c$, are reported in Table 6 .

\begin{tabular}{|c|c|c|c|c|c|c|c|c|}
\hline & \multicolumn{2}{|c|}{$c=1$} & \multicolumn{2}{|c|}{$c=1 / 2$} & \multicolumn{2}{|c|}{$c=1 / 4$} & \multicolumn{2}{|c|}{$c=1 / 8$} \\
\hline & $\begin{array}{l}\operatorname{Re}\left(z_{0}\right) \\
\operatorname{Im}\left(z_{0}\right)\end{array}$ & $\begin{array}{l}\approx-0.55 \\
\approx \pm 0.40\end{array}$ & $\begin{array}{l}\operatorname{Re}\left(z_{0}\right) \\
\operatorname{Im}\left(z_{0}\right)\end{array}$ & $\begin{array}{l}\approx-0.72 \\
\approx \pm 0.24\end{array}$ & $\begin{array}{l}\operatorname{Re}\left(z_{0}\right) \\
\operatorname{Im}\left(z_{0}\right)\end{array}$ & $\begin{array}{l}\approx-0.77 \\
\approx \pm 0.12\end{array}$ & $\begin{array}{l}\operatorname{Re}\left(z_{0}\right) \\
\operatorname{Im}\left(z_{0}\right)\end{array}$ & $\begin{array}{l}\approx-0.78 \\
\approx \pm 0.06\end{array}$ \\
\hline $2 n$ & $G L_{2 n}$ & $q=50$ & $G L_{2 n}$ & $q=50$ & $G L_{2 n}$ & $q=50$ & $G L_{2 n}$ & $q=50$ \\
\hline 8 & $6.47-04$ & $3.19-04$ & $2.42-02$ & $2.49-03$ & $2.43-01$ & $8.93-03$ & $6.03-01$ & $8.46-02$ \\
\hline 16 & $4.70-08$ & $1.16-07$ & $1.12-04$ & $4.83-07$ & $1.78-02$ & $1.10-04$ & $7.86-02$ & $2.77-03$ \\
\hline 32 & $1.57-14$ & $1.91-14$ & $9.55-09$ & $7.54-13$ & $2.37-05$ & $9.82-10$ & $2.56-02$ & $1.63-07$ \\
\hline 64 & -- & -- & -- & -- & $7.15-09$ & -- & $1.19-04$ & $3.17-14$ \\
\hline 128 & -- & -- & -- & -- & -- & -- & $1.46-09$ & -- \\
\hline
\end{tabular}

Table 6. Relative errors in $I_{5}=\int_{-1.5}^{0}\left[(c \sin (x))^{2}+(\cos (x)+\sin (x))^{2}\right]^{-3 / 2} d x$. 
Also integrals of the form

$$
I_{6}=\int_{\varepsilon}^{1} \frac{f(x)}{x} d x
$$

with $f(x)$ smooth and $\varepsilon>0$ small, can be efficiently evaluated using a GaussLegendre rule, after having introduced the change of variable $x=t^{q}$. Some relative errors concerning an integral of this type, with $f(x)=e^{x}$, are reported in Table 7.

\begin{tabular}{|c||c|c||c|c||c|c|}
\hline \multicolumn{1}{|c||}{} & \multicolumn{2}{c||}{$\varepsilon=10^{-1}$} & \multicolumn{2}{c||}{$\varepsilon=10^{-3}$} & \multicolumn{2}{c|}{$\varepsilon=10^{-5}$} \\
\hline$n$ & $q=1$ & $q=3$ & $q=1$ & $q=6$ & $q=1$ & $q=8$ \\
\hline 4 & $4.44-02$ & $2.36-06$ & $3.38-01$ & $2.37-04$ & $5.73-01$ & $1.03-03$ \\
8 & $2.48-05$ & $2.75-12$ & $1.96-01$ & $8.47-09$ & $4.74-01$ & $2.94-07$ \\
16 & $7.15-10$ & -- & $7.45-02$ & -- & $3.71-01$ & -- \\
32 & -- & -- & $1.09-02$ & -- & $2.66-01$ & -- \\
64 & -- & -- & $2.05-04$ & -- & $1.64-01$ & -- \\
128 & -- & -- & $6.43-08$ & -- & $7.37-02$ & -- \\
256 & -- & -- & -- & -- & $1.60-02$ & -- \\
\hline
\end{tabular}

Table 7. Relative errors in $I_{6}=\int_{\varepsilon}^{1} \frac{1}{q} \frac{q e^{t^{q}}}{t} d t$.

In the introduction we have remarked that for small values of $n$, the approach we have proposed gives slightly better results than the DE-rule. This is due to the fact that the stepsize $h$ of the DE-rule is chosen to optimize the rate of convergence, that is, the behavior of the remainder term as $n \rightarrow \infty$ (see [15]). Indeed, as $n \rightarrow \infty$ the accuracy given by the DE-rule is superior to that given by our numerical integration approach.

However, in the applications we are interested in, we ought to achieve a good accuracy by taking a small value of $n$. Therefore, in the case of the DErule, for a given (low) value of $n$ we would like to choose the parameter $h$ which minimizes the error.

For the integrals $I_{1}$ through $I_{5}$ of this section we have plotted the behavior of the error term as a function of $h$, for several values of $n$. In all cases we have examined, a proper choice of $h$ has reduced substantially the error. Here we report a few examples, which are however representative of all cases we have examined. In all cases there exists an optimal choice of $h$ which allows to reach very high accuracies using a small value of $n$. However we do not know a simple formula to compute such value, or a good approximation of it. We note that all figures have been obtained by subdividing the domain of $h$ into 100 equal parts. Therefore the actual minima could be even smaller than those appearing in the figures. 


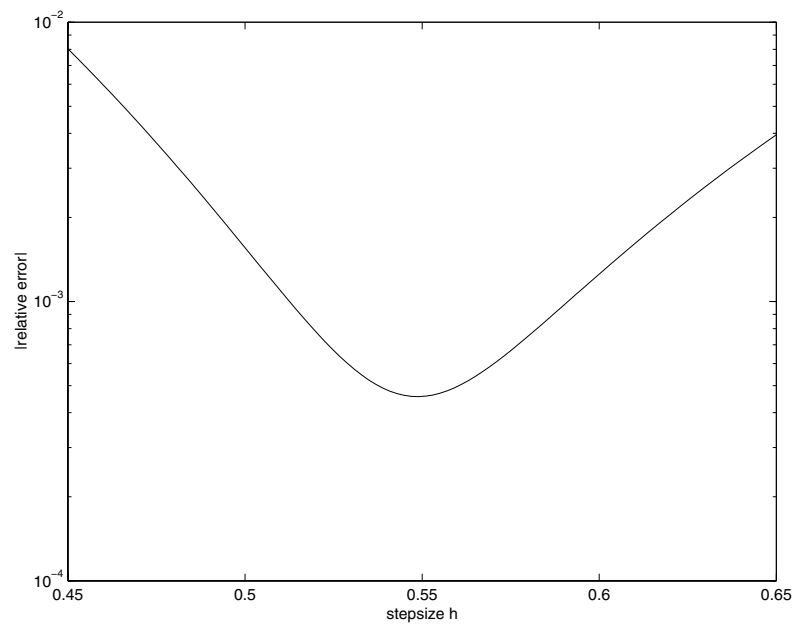

Figure 1. Integral $I_{1}, n=3, h \in(0.45,0.65)$.

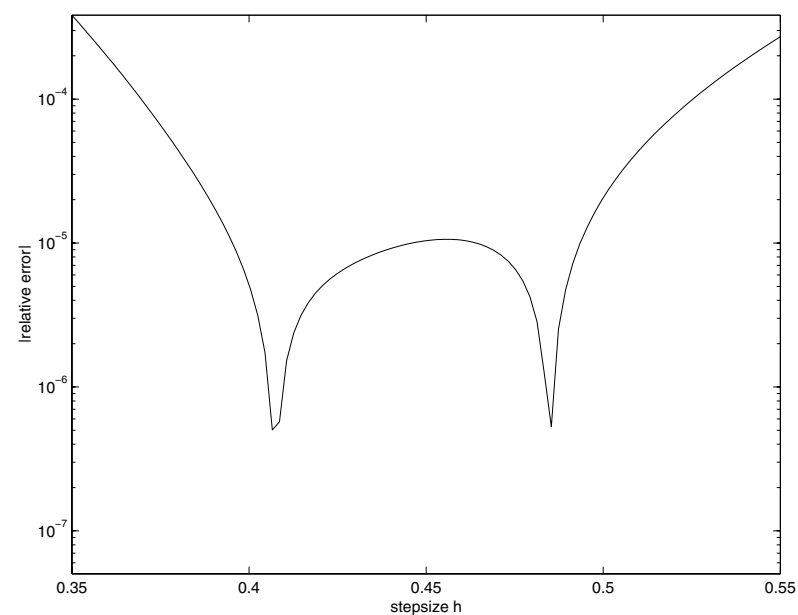

Figure 2. Integral $I_{1}, n=5, h \in(0.35,0.55)$. 


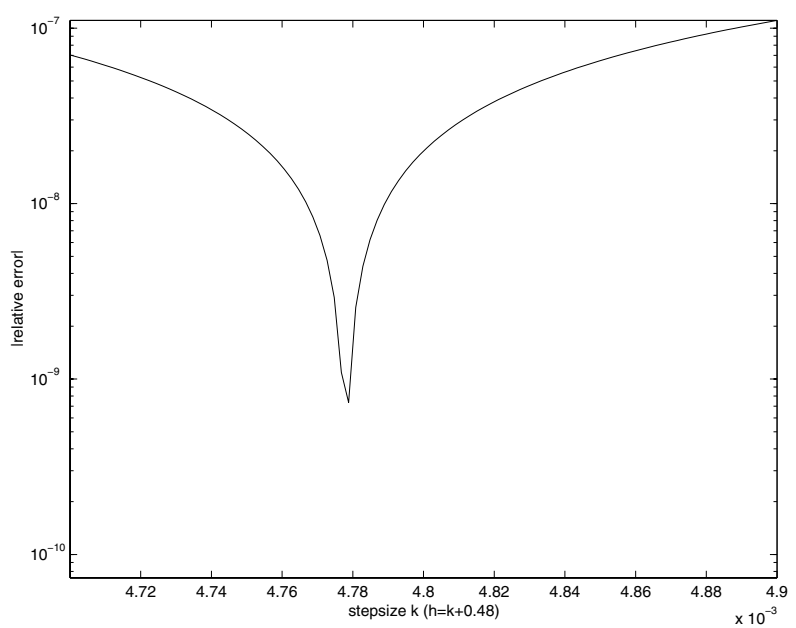

Figure 3. Integral $I_{1}, n=5, h \in(0.4847,0.4849)$.

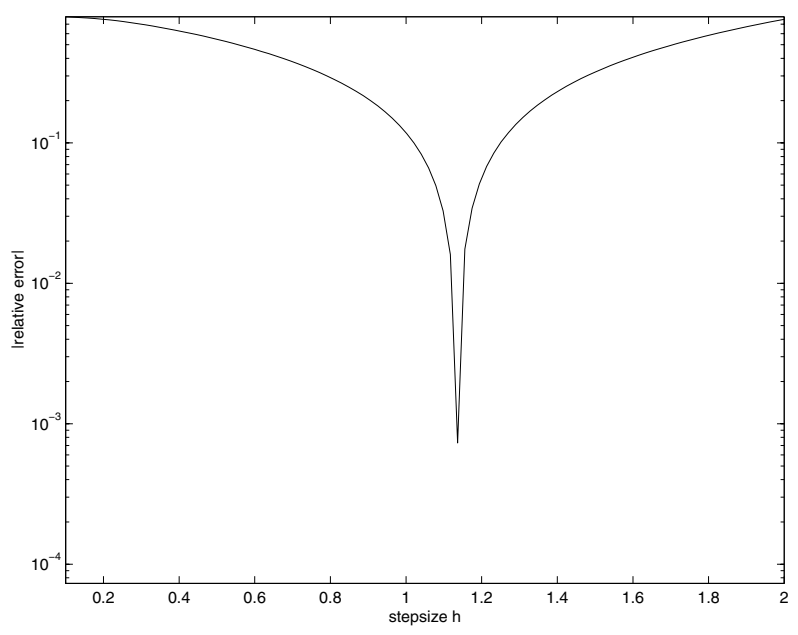

Figure 4. Integral $I_{4}, r=0.5, \varepsilon=10^{-1}, n=3, h \in(0.1,2)$. 


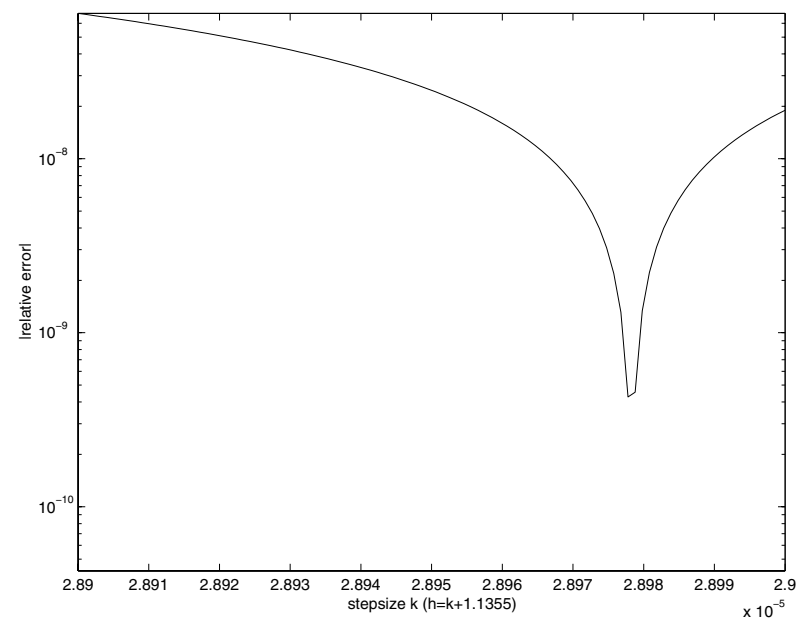

Figure 5. Integral $I_{4}, r=0.5, \varepsilon=10^{-1}, n=3, h \in(1.1355289,1.135529)$.

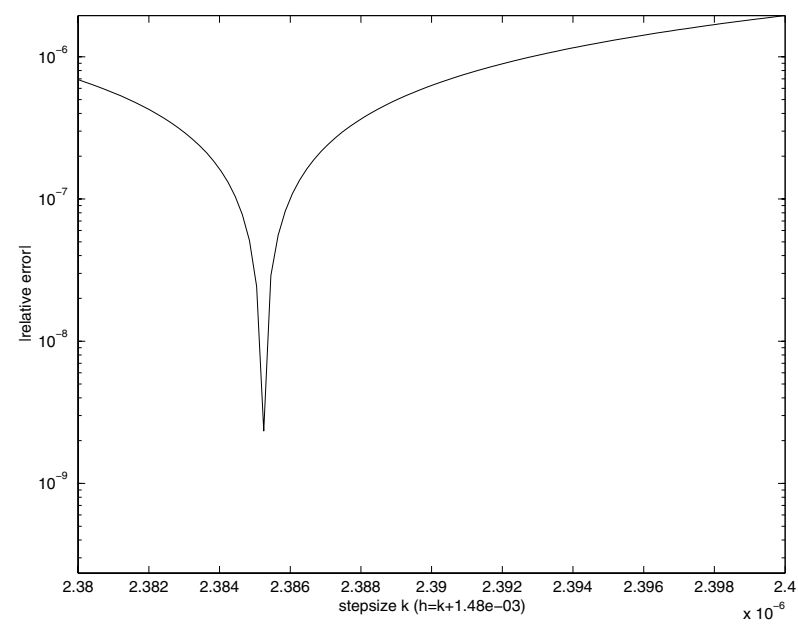

Figure 6. Integral $I_{4}, r=0.5, \varepsilon=10^{-3}, n=3, h \in(1.48238 e-03,1.4824 e-03)$. 


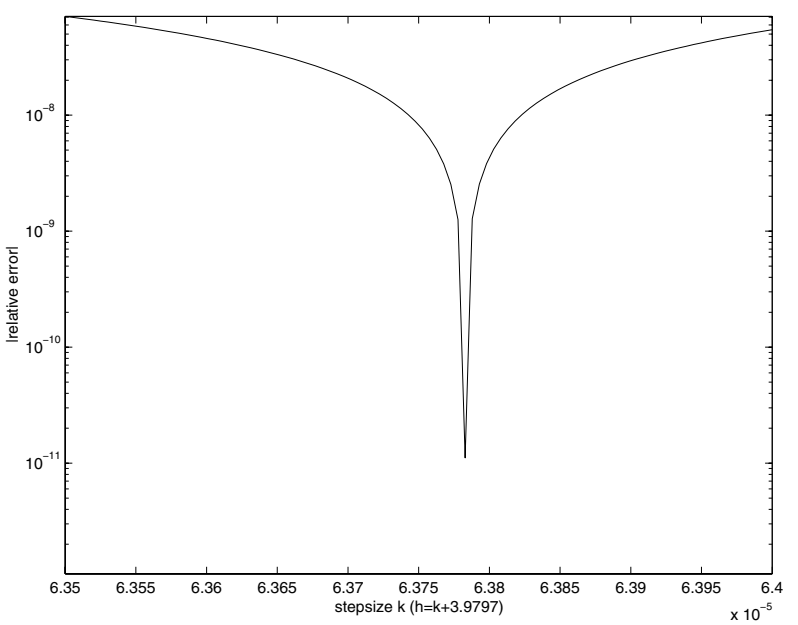

Figure 7. Integral $I_{5}, \varepsilon=1 / 2, n=3, h \in(3.9797635,3.979764)$.

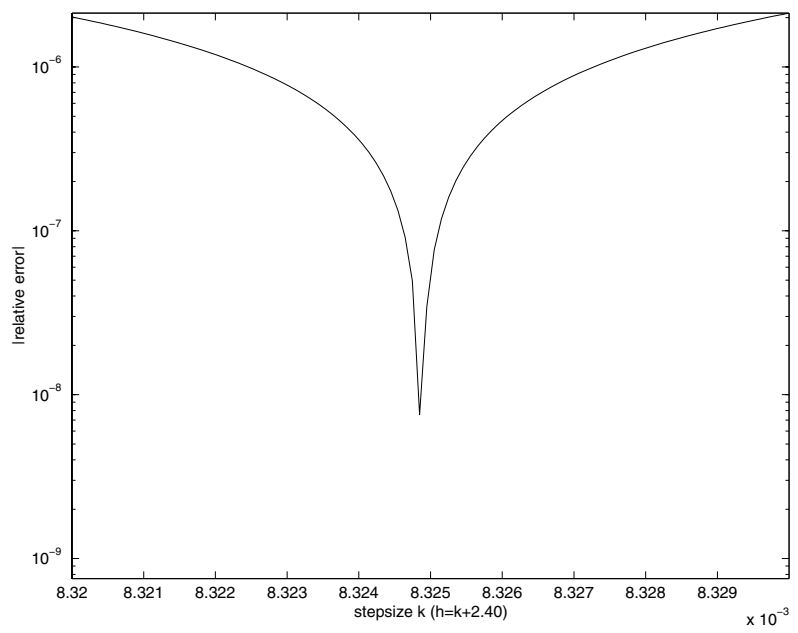

Figure 8. Integral $I_{5}, \varepsilon=1 / 8, n=3, h \in(2.40832,2.40833)$. 
The last integral we need to consider is

$$
I_{7}=f_{0}^{R(y)} \frac{f(x)}{x} d x
$$

defined as Hadamard finite-part, where $y$ is an outer variable, which can make $R(y)$ very close to zero. Since a change of variable does not leave unchanged the value of the integral, and we do not want to construct an ad hoc rule for each value of the variable $y$, we suggest to proceed as follows. If $f(0)$ exists and can be easily computed, then write

$$
f_{0}^{R(y)} \frac{f(x)}{x} d x=\int_{0}^{R(y)} \frac{f(x)-f(0)}{x} d x+f(0) \log R(y)
$$

and apply the Gauss-Legendre to the integral on the right-hand side of (2.15). If however this is not the case, then write

$$
f_{0}^{R(y)} \frac{f(x)}{x} d x=\left(f_{0}^{r}+\int_{r}^{R(y)}\right) \frac{f(x)}{x} d x .
$$

where $r>0$ has a suitable fixed value. To compute the first integral on the right-hand side we use a standard product rule for Hadamard finite part integrals, whose coefficients have been computed once for all ([7]). The second integral can be computed by the technique suggested for (2.13) with a large $q$ (for example, $q=100$ ) when $f$ is smooth. The choice of $r$ may depend on $f$. Indeed, if $f$ has a pair of complex poles with the real part fixed, independent of $y$ and belonging to the interval of integration $(0, R(y))$, it is convenient to take $r$ equal to the real part of the poles. If the real part varies with $y$ in a little range of $(0, R(y))$ we can choose $r$ as an intermediate value of the range; otherwise, it is convenient to use a product formula without splitting. Finally, if $f$ is smooth, we suggest to choose $r$ small.

In the Tables 8 and 9 we have reported the relative errors obtained first by applying directly the product rule with $n$ quadrature points $\left(P R_{n}\right)$ to $I_{5}$ in (2.14), and then by splitting $I_{5}$ as in (2.16) and computing the integrals to the left-hand side as suggested above $\left(P R_{n}+G L_{n}\right)$. In particular, in Table 8 we report the relative errors corresponding to the choice $f(x)=f_{1}(x)=1 /\left[(x-r)^{2}+\varepsilon^{2}\right]$ with $r=0.1$ and $\varepsilon$ varying; in Table 9 we give those corresponding to $f(x)=f_{2}(x)=e^{x}$ and $r=0.1$ in (2.16). 


\begin{tabular}{|c||c|c||c|c||c|c|}
\hline \multicolumn{1}{|c||}{} & \multicolumn{2}{c||}{$\varepsilon=10^{-1}$} & \multicolumn{2}{c||}{$\varepsilon=10^{-2}$} & \multicolumn{2}{c|}{$\varepsilon=10^{-3}$} \\
\hline $2 n$ & $P R_{2 n}$ & $P R_{n}+G L_{n}$ & $P R_{2 n}$ & $P R_{n}+G L_{n}$ & $P R_{2 n}$ & $P R_{n}+G L_{n}$ \\
\hline 4 & $1.83+00$ & $2.31-01$ & $1.00+00$ & $4.72-01$ & $1.00+00$ & $8.35-01$ \\
8 & $2.45+00$ & $1.50-01$ & $3.15+00$ & $1.03+00$ & $1.27+00$ & $4.17-01$ \\
16 & $9.62-02$ & $2.86-04$ & $1.06+01$ & $6.54-02$ & $6.66+00$ & $9.01-01$ \\
32 & $6.53-05$ & $9.92-10$ & $6.86+00$ & $7.60-03$ & $9.63+00$ & $1.07+00$ \\
64 & $1.30-11$ & $2.32-13$ & $1.66+00$ & $1.51-05$ & $9.03+00$ & $1.64-01$ \\
128 & -- & -- & $1.42-01$ & $3.33-12$ & $3.67+01$ & $2.31-03$ \\
256 & -- & -- & $4.54-04$ & -- & $4.45+01$ & $5.77-07$ \\
512 & -- & -- & $9.03-10$ & -- & $1.22+00$ & $3.86-15$ \\
\hline
\end{tabular}

Table 8. Relative errors in $I_{7}=\int_{0}^{R(y)} \frac{\left[(x-0.1)^{2}+\varepsilon^{2}\right]^{-1}}{x} d x$ $=\left(\int_{0}^{r}+\int_{r}^{R(y)}\right) \frac{\left[(x-0.1)^{2}+\varepsilon^{2}\right]^{-1}}{x} d x$ with $r=0.1, R(y)=0.5$.

\begin{tabular}{|c||c|c||c|c|}
\hline \multicolumn{1}{|c||}{} & \multicolumn{2}{c||}{$R(y)=10^{-2}$} & \multicolumn{2}{c|}{$R(y)=0.5$} \\
\hline $2 n$ & $P R_{2 n}$ & $P R_{n}+G L_{n}$ & $P R_{2 n}$ & $P R_{n}+G L_{n}$ \\
\hline 4 & $1.14-11$ & $1.13-03$ & $1.80-03$ & $1.97-02$ \\
8 & -- & $1.29-07$ & $4.69-10$ & $6.49-06$ \\
16 & -- & $6.38-15$ & $4.09-13$ & $1.20-12$ \\
32 & -- & -- & -- & $3.65-13$ \\
64 & -- & -- & -- & -- \\
\hline
\end{tabular}

Table 9. Relative errors in $I_{7}=\int_{0}^{R(y)} \frac{e^{x}}{x} d x=\int_{0}^{r} \frac{e^{x}}{x} d x$ $+\int_{r^{\frac{1}{q}}}^{(R(y))^{\frac{1}{q}}} \frac{q e^{t^{q}}}{t} d t$ with $r=0.1, q=100$.

\section{$\S 3 . \quad$ The Four-dimensional Integral}

The integral we have to compute has the following form

$$
I_{\Delta}=f_{\Delta} d \bar{x} f_{\Delta} \frac{\bar{N}_{i}(\bar{x}) \bar{N}_{j}(\bar{y})}{\|\bar{x}-\bar{y}\|^{3}} d \bar{y}
$$

where for simplicity we assume $\Delta$ to be a triangle with corners at $(0,0),\left(a_{1}, a_{2}\right)$, $\left(b_{1}, b_{2}\right)$ and $\left\{\bar{N}_{i}(\bar{x})\right\},\left\{\bar{N}_{j}(\bar{y})\right\}$ are the shape and the test functions, respectively. Our approach however easily extends to the case of a curved triangle, given by a smooth parametric representation.

By the linear transformation $\bar{x}=A x, \bar{y}=A y$ with

$$
A=\left(\begin{array}{ll}
a_{1} & b_{1} \\
a_{2} & b_{2}
\end{array}\right)
$$

we map the domain $\Delta$ into the reference triangle $T$ having corners at $(0,0)$, $(1,0)(0,1)$. In this case the integral $(3.1)$ can be replaced by 


$$
I_{T}=|A|^{2} f_{T} d x f_{T} \frac{N_{i}(x) N_{j}(y)}{\|A(x-y)\|^{3}} d y,
$$

where $|A|=\operatorname{det}(A), N_{i}(x):=\bar{N}_{i}(A x)$ and $N_{j}(y):=\bar{N}_{j}(A y)$.

The value of $I_{T}$ is generally different from that of $I_{\Delta}$; however the sum of the values of the integrals defined over all boundary elements $\Delta_{i}$ is equal to the sum of the corresponding integrals of form (3.2). Therefore from now on we refer to the form (3.2).

To compute (3.2) we split it as follows:

$$
\begin{aligned}
& I_{T}=|A|^{2} {\left[\int_{T} N_{i}(x) d x f_{T} \frac{N_{j}(y)-N_{j}(x)}{\|A(x-y)\|^{3}} d y\right.} \\
&\left.+\int_{T} N_{i}(x) N_{j}(x) d x f \frac{d y}{\|A(x-y)\|^{3}}\right] \\
&=:|A|^{2}\left[I_{T, 1}+I_{T, 2}\right]
\end{aligned}
$$

since the inner integral in $I_{T, 2}$ can be evaluated analytically and the strong singularity in $I_{T, 1}$ is of Cauchy type.

As regards $I_{T, 1}$, first we introduce the following polar coordinates

$$
\left\{\begin{array}{l}
y_{1}=x_{1}+r \cos (\vartheta) \\
y_{2}=x_{2}+r \sin (\vartheta)
\end{array}\right.
$$

where $0 \leq r \leq R(\vartheta), 0 \leq \vartheta \leq 2 \pi$. Then we split the interval $(0,2 \pi)$ into three subintervals $\left(\vartheta_{0}, \vartheta_{1}\right),\left(\vartheta_{1}, \vartheta_{2}\right)$ and $\left(\vartheta_{2}, \vartheta_{3}\right)$, with

$$
\left\{\begin{array}{l}
\vartheta_{0}=-\pi+\arctan \left(\frac{x_{2}}{x_{1}}\right) \\
\vartheta_{1}=-\arctan \left(\frac{x_{2}}{1-x_{1}}\right) \\
\vartheta_{2}=\pi-\arctan \left(\frac{1-x_{2}}{x_{1}}\right) \\
\vartheta_{3}=\pi+\arctan \left(\frac{x_{2}}{x_{1}}\right) .
\end{array}\right.
$$

For $\vartheta \in\left[\vartheta_{l-1}, \vartheta_{l}\right], l=1,2,3$, we have $0 \leq r \leq R_{l}$ with

$$
R_{l}:=R_{l}\left(x_{1}, x_{2}, \vartheta\right)=\frac{d_{l}\left(x_{1}, x_{2}\right)}{p_{l}(\vartheta)}
$$


where

$$
\left\{\begin{array} { l } 
{ d _ { 1 } ( x _ { 1 } , x _ { 2 } ) = x _ { 2 } } \\
{ d _ { 2 } ( x _ { 1 } , x _ { 2 } ) = \frac { 1 } { \sqrt { 2 } } ( 1 - x _ { 1 } - x _ { 2 } ) , } \\
{ d _ { 3 } ( x _ { 1 } , x _ { 2 } ) = x _ { 1 } }
\end{array} \quad \left\{\begin{array}{l}
p_{1}(\vartheta)=-\sin (\vartheta) \\
p_{2}(\vartheta)=\frac{1}{\sqrt{2}}[\sin (\vartheta)+\cos (\vartheta)] . \\
p_{3}(\vartheta)=-\cos (\vartheta)
\end{array}\right.\right.
$$

Our integral $I_{T, 1}$ can then be expressed as follows:

$$
\begin{aligned}
& I_{T, 1}=\int_{0}^{1} d x_{1} \int_{0}^{1-x_{1}} N_{i}\left(x_{1}, x_{2}\right) d x_{2} \\
& \quad \sum_{l=1}^{3} \int_{\vartheta_{l-1}}^{\vartheta_{l}} d \vartheta f_{0}^{R_{l}} \frac{N_{j}\left(x_{1}+r \cos (\vartheta), x_{2}+r \sin (\vartheta)\right)-N_{j}\left(x_{1}, x_{2}\right)}{r^{2} f(\vartheta)} d r
\end{aligned}
$$

where

$$
f(\vartheta)=\left[\left(a_{1} \cos (\vartheta)+b_{1} \sin (\vartheta)\right)^{2}+\left(a_{2} \cos (\vartheta)+b_{2} \sin (\vartheta)\right)^{2}\right]^{3 / 2} .
$$

Notice that $f(\vartheta)$ vanishes when

$$
\tan (\vartheta)=\alpha \pm i \beta
$$

with

$$
\alpha=-\frac{a_{1} b_{1}+a_{2} b_{2}}{b_{1}^{2}+b_{2}^{2}}, \quad \beta=\frac{a_{2} b_{1}-a_{1} b_{2}}{b_{1}^{2}+b_{2}^{2}} .
$$

In particular the poles of $f(\vartheta)$ depend on the corners of the triangle $\Delta$. Therefore their distance from the interval of integration with respect to the variable $\vartheta$ depends upon the shape of $\Delta$ and could be very small. For example, in the case of a triangle $\Delta$, whose corners are at $(0,0),(a, 1),(0,1)$, we have approximately the following complex poles: $(-1.01+k \pi) \pm 0.40 \imath$ when $a=1$, $(-0.85+k \pi) \pm 0.24 \imath$ when $a=1 / 2$ and $(-0.80+k \pi) \pm 0.12 \imath$ when $a=1 / 4$, $k \in \mathbb{Z}$.

To proceed we set

$$
N_{j}^{r}\left(x_{1}, x_{2} ; r, \vartheta\right)=\frac{N_{j}\left(x_{1}+r \cos (\vartheta), x_{2}+r \sin (\vartheta)\right)-N_{j}\left(x_{1}, x_{2}\right)}{r}
$$


and write

$$
\begin{aligned}
I_{T, 1}= & \int_{0}^{1} d x_{1} \int_{0}^{1-x_{1}} N_{i}\left(x_{1}, x_{2}\right) d x_{2} \\
& \sum_{l=1}^{3} \int_{\vartheta_{l-1}}^{\vartheta_{l}}[f(\vartheta)]^{-1} d \vartheta f_{0}^{R_{l}} \frac{N_{j}^{r}\left(x_{1}, x_{2} ; r, \vartheta\right)}{r} d r \\
= & \int_{0}^{1} d x_{1} \int_{0}^{1-x_{1}} N_{i}\left(x_{1}, x_{2}\right) d x_{2} \sum_{l=1}^{3} \int_{\vartheta_{l-1}}^{\vartheta_{l}}[f(\vartheta)]^{-1} \\
& {\left[\int_{0}^{R_{l}} \frac{N_{j}^{r}\left(x_{1}, x_{2} ; r, \vartheta\right)-N_{j}^{r}\left(x_{1}, x_{2} ; 0, \vartheta\right)}{r} d r+N_{j}^{r}\left(x_{1}, x_{2} ; 0, \vartheta\right) \log \left(R_{l}\right)\right] d \vartheta }
\end{aligned}
$$

Remark. Notice that in the case of $\left\{N_{j}\right\}$ linear, that is $N_{j}=N_{j}^{l}, j=$ $1,2,3$, and

$$
\begin{aligned}
& N_{1}^{l}\left(x_{1}, x_{2}\right)=x_{1}, \\
& N_{2}^{l}\left(x_{1}, x_{2}\right)=x_{2}, \\
& N_{3}^{l}\left(x_{1}, x_{2}\right)=1-x_{1}-x_{2},
\end{aligned}
$$

we have

$$
\begin{aligned}
& N_{1}^{r}\left(x_{1}, x_{2} ; r, \vartheta\right)=\cos (\vartheta), \\
& N_{2}^{r}\left(x_{1}, x_{2} ; r, \vartheta\right)=\sin (\vartheta), \\
& N_{3}^{r}\left(x_{1}, x_{2} ; r, \vartheta\right)=-[\sin (\vartheta)+\cos (\vartheta)] .
\end{aligned}
$$

Therefore $N_{j}^{r}$ depends only upon $\vartheta$ and the integral over $\left(0, R_{l}\right)$ in the first expression of $I_{T, 1}$ in (3.9) can be performed analytically.

By denoting

$$
D_{j}\left(x_{1}, x_{2} ; r, \vartheta\right):=\frac{N_{j}^{r}\left(x_{1}, x_{2} ; r, \vartheta\right)-N_{j}^{r}\left(x_{1}, x_{2} ; 0, \vartheta\right)}{r},
$$

we write

$$
I_{T, 1}=\sum_{l=1}^{3} I_{T, 1}^{l},
$$


where

$$
\begin{aligned}
I_{T, 1}^{l}= & \int_{0}^{1} d x_{1} \int_{0}^{1-x_{1}} N_{i}\left(x_{1}, x_{2}\right) d x_{2} \int_{\vartheta_{l-1}}^{\vartheta_{l}}[f(\vartheta)]^{-1} \\
& {\left[\int_{0}^{R_{l}} D_{j}\left(x_{1}, x_{2} ; r, \vartheta\right) d r+N_{j}^{r}\left(x_{1}, x_{2} ; 0, \vartheta\right) \log \left(R_{l}\right)\right] d \vartheta, l=1,2,3 . }
\end{aligned}
$$

Remark. In the case of $\left\{N_{j}\right\}$ quadratic, that is $N_{j}=N_{j}^{q}, j=1, \ldots, 6$, and

$$
\begin{aligned}
& N_{1}^{q}\left(x_{1}, x_{2}\right)=x_{1}\left(2 x_{1}-1\right), \\
& N_{2}^{q}\left(x_{1}, x_{2}\right)=x_{2}\left(2 x_{2}-1\right), \\
& N_{3}^{q}\left(x_{1}, x_{2}\right)=\left(1-x_{1}-x_{2}\right)\left(2\left(1-x_{1}-x_{2}\right)-1\right), \\
& N_{4}^{q}\left(x_{1}, x_{2}\right)=4 x_{1} x_{2}, \\
& N_{5}^{q}\left(x_{1}, x_{2}\right)=4 x_{2}\left(1-x_{1}-x_{2}\right), \\
& N_{6}^{q}\left(x_{1}, x_{2}\right)=4 x_{1}\left(1-x_{1}-x_{2}\right),
\end{aligned}
$$

we have

$$
\begin{aligned}
& D_{1}\left(x_{1}, x_{2} ; r, \vartheta\right)=2 \cos ^{2}(\vartheta), \\
& D_{2}\left(x_{1}, x_{2} ; r, \vartheta\right)=2 \sin ^{2}(\vartheta), \\
& D_{3}\left(x_{1}, x_{2} ; r, \vartheta\right)=2[\sin (\vartheta)+\cos (\vartheta)]^{2}, \\
& D_{4}\left(x_{1}, x_{2} ; r, \vartheta\right)=4 \sin (\vartheta) \cos (\vartheta), \\
& D_{5}\left(x_{1}, x_{2} ; r, \vartheta\right)=-4 \sin (\vartheta)[\sin (\vartheta)+\cos (\vartheta)], \\
& D_{6}\left(x_{1}, x_{2} ; r, \vartheta\right)=-4 \cos (\vartheta)[\sin (\vartheta)+\cos (\vartheta)] .
\end{aligned}
$$

Therefore, since $D_{j}\left(x_{1}, x_{2} ; r, \vartheta\right)$ does not depend upon $r$, the integral over $\left(0, R_{l}\right)$ in $(3.11)$ can be performed analytically.

Since $R_{l}$ takes different values for $l=1,2,3$, the computation of $I_{T, 1}^{l}$ have to be performed using different strategies according to these values.

We first consider $I_{T, 1}^{1}$, where we have set $x_{2}=\left(1-x_{1}\right) \tilde{x}_{2}, \tilde{x}_{2} \in(0,1)$ :

$$
\begin{aligned}
I_{T, 1}^{1} & =\int_{0}^{1}\left(1-x_{1}\right) d x_{1} \int_{0}^{1} N_{i}\left(x_{1},\left(1-x_{1}\right) \tilde{x}_{2}\right) d \tilde{x}_{2} \int_{\tilde{\vartheta}_{0}}^{\tilde{\vartheta}_{1}}[f(\vartheta)]^{-1} \\
& {\left[\int_{0}^{\tilde{R}_{1}} D_{j}\left(x_{1},\left(1-x_{1}\right) \tilde{x}_{2} ; r, \vartheta\right) d r+N_{j}^{r}\left(x_{1},\left(1-x_{1}\right) \tilde{x}_{2} ; 0, \vartheta\right) \log \left(\tilde{R}_{1}\right)\right] d \vartheta }
\end{aligned}
$$


with

$$
\left\{\begin{array}{l}
\tilde{\vartheta}_{0}=-\pi+\arctan \left(\frac{\left(1-x_{1}\right) \tilde{x}_{2}}{x_{1}}\right) \\
\tilde{\vartheta}_{1}=-\arctan \left(\tilde{x}_{2}\right) \\
\tilde{R}_{1}=\frac{\left(1-x_{1}\right) \tilde{x}_{2}}{-\sin (\vartheta)}
\end{array}\right.
$$

Taking into account of the behaviors of the integrand functions of each integral in (3.13) and of the behaviors of the endpoints $\tilde{\vartheta}_{0}, \tilde{\vartheta}_{1}$ as $x_{1}, \tilde{x}_{2} \rightarrow 0,1$, the following smoothing transformations have been introduced

$$
\left\{\begin{array}{l}
x_{1}=\gamma_{0,1}^{q_{11}}(x), x \in(0,1), \\
\tilde{x}_{2}=\gamma_{0}^{q_{12}}(y), y \in(0,1), \\
\vartheta=\gamma_{0}^{q_{13}}((b-a) t+a), t \in(0,1),
\end{array}\right.
$$

whose smoothing exponents $q_{1 l}, l=1,2,3$, will be specified later. In the last change of variable $(a, b)$ denotes the interval of integration with respect to the variable $\vartheta$. If no real parts $\vartheta_{R}$ of the poles of $f(\vartheta)$ belong to $\left(\tilde{\vartheta}_{0}, \tilde{\vartheta}_{1}\right)$, the interval of integration is $\left(\tilde{\vartheta}_{0}, \tilde{\vartheta}_{1}\right)$. Otherwise we split this interval into two subintervals $\left(\tilde{\vartheta}_{0}, \vartheta_{R}\right)$ and $\left(\vartheta_{R}, \tilde{\vartheta}_{1}\right)$ and we apply the above change of variable to each of them, taking $(a, b) \equiv\left(\tilde{\vartheta}_{0}, \vartheta_{R}\right)$ and $(a, b) \equiv\left(\vartheta_{R}, \tilde{\vartheta}_{1}\right)$.

Finally, for the variables $x_{1}$ and $\tilde{x_{2}}$ the numerical integration is performed using the $n$-point Gauss-Legendre rule, while for the variable $\vartheta$ we have used the $2 n$-point Gauss-Legendre rule.

In the case of $I_{T, 1}^{2}$ we have

$$
\begin{aligned}
I_{T, 1}^{2} & =\int_{0}^{1}\left(1-x_{1}\right) d x_{1} \int_{0}^{1} N_{i}\left(x_{1},\left(1-x_{1}\right) \tilde{x}_{2}\right) d \tilde{x}_{2} \int_{\tilde{\vartheta}_{1}}^{\tilde{\vartheta}_{2}}[f(\vartheta)]^{-1} \\
& {\left[\int_{0}^{\tilde{R}_{2}} D_{j}\left(x_{1},\left(1-x_{1}\right) \tilde{x}_{2} ; r, \vartheta\right) d r+N_{j}^{r}\left(x_{1},\left(1-x_{1}\right) \tilde{x}_{2} ; 0, \vartheta\right) \log \left(\tilde{R}_{2}\right)\right] d \vartheta }
\end{aligned}
$$

where

$$
\left\{\begin{array}{l}
\tilde{\vartheta}_{1}=-\arctan \left(\tilde{x}_{2}\right) \\
\tilde{\vartheta}_{2}=\pi-\arctan \left(\frac{1-\left(1-x_{1}\right) \tilde{x}_{2}}{x_{1}}\right) \\
\tilde{R}_{2}=\frac{\left(1-x_{1}\right)\left(1-\tilde{x}_{2}\right)}{\sin (\vartheta)+\cos (\vartheta)} .
\end{array}\right.
$$


In (3.14) we first introduce the smoothing transformations

$$
\left\{\begin{array}{l}
x_{1}=\gamma_{0,1}^{q_{21}}(x), x \in(0,1), \\
\tilde{x}_{2}=\gamma_{1}^{q_{22}}(y), y \in(0,1), \\
\vartheta=\gamma_{0,1}^{q_{23}}((b-a) t+a), t \in(0,1),
\end{array}\right.
$$

where, as above, $(a, b) \equiv\left(\tilde{\vartheta}_{1}, \tilde{\vartheta}_{2}\right)$ is split into $\left(\tilde{\vartheta}_{1}, \vartheta_{R}\right) \cup\left(\vartheta_{R}, \tilde{\vartheta}_{2}\right)$ whenever there exists a pole of $f(\vartheta)$ whose real part $\vartheta_{R} \in\left(\tilde{\vartheta}_{1}, \tilde{\vartheta}_{2}\right)$. Then we apply the same Gauss-Legendre product rule used in the previous case.

The integral

$$
\begin{aligned}
I_{T, 1}^{3} & =\int_{0}^{1}\left(1-x_{1}\right) d x_{1} \int_{0}^{1} N_{i}\left(x_{1},\left(1-x_{1}\right) \tilde{x}_{2}\right) d \tilde{x}_{2} \int_{\tilde{\vartheta}_{2}}^{\tilde{\vartheta}_{3}}[f(\vartheta)]^{-1} \\
& {\left[\int_{0}^{\tilde{R}_{3}} D_{j}\left(x_{1},\left(1-x_{1}\right) \tilde{x}_{2} ; r, \vartheta\right) d r+N_{j}^{r}\left(x_{1},\left(1-x_{1}\right) \tilde{x}_{2} ; 0, \vartheta\right) \log \left(\tilde{R}_{3}\right)\right] d \vartheta }
\end{aligned}
$$

where

$$
\left\{\begin{array}{l}
\tilde{\vartheta}_{2}=\pi-\arctan \left(\frac{1-\left(1-x_{1}\right) \tilde{x}_{2}}{x_{1}}\right), \\
\tilde{\vartheta}_{3}=\pi+\arctan \left(\frac{\left(1-x_{1}\right) \tilde{x}_{2}}{x_{1}}\right), \\
\tilde{R}_{3}=\frac{x_{1}}{-\cos (\vartheta)},
\end{array}\right.
$$

is treated similarly to the previous cases. Here we set

$$
\left\{\begin{array}{l}
x_{1}=\gamma_{0}^{q_{31}}(x), x \in(0,1), \\
\tilde{x}_{2}=\gamma_{0,1}^{q_{32}}(y), y \in(0,1), \\
\vartheta=\gamma_{0,1}^{q_{33}}((b-a) t+a), t \in(0,1),
\end{array}\right.
$$

and the interval $(a, b) \equiv\left(\tilde{\vartheta}_{2}, \tilde{\vartheta}_{3}\right)$ is split into $\left(\tilde{\vartheta}_{2}, \vartheta_{R}\right) \cup\left(\vartheta_{R}, \tilde{\vartheta}_{3}\right)$ if $\vartheta_{R} \in\left(\tilde{\vartheta}_{2}, \tilde{\vartheta}_{3}\right)$.

In the case of linear and quadratic functions $N_{j}$, a choice of the above smoothing exponents $q_{l 1}, q_{l 2}, q_{l 3}, l=1,2,3$, which leads to very satisfactory results, is reported in Table 10 .

However, for basis functions of higher order the choice

$$
q_{l 1}=q_{l 2}=q_{l 3}=2, l=1,2,3,
$$

is quite satisfactory.

As already stated earlier, the inner most integral in $I_{T, 2}$ defined in (3.3), can be evaluated analytically. Indeed, we have 


\begin{tabular}{||c|c||c|c|c||c|c|c||c|c|c||}
\hline \multicolumn{3}{||c||}{$N_{i}, N_{j}$ linear } & \multicolumn{3}{c||}{$I_{T, 1}^{1}$} & \multicolumn{3}{c||}{$I_{T, 1}^{2}$} & \multicolumn{3}{||}{$I_{T, 1}^{3}$} \\
\hline$i$ & $j$ & $q_{11}$ & $q_{12}$ & $q_{13}$ & $q_{21}$ & $q_{22}$ & $q_{23}$ & $q_{31}$ & $q_{32}$ & $q_{33}$ \\
\hline 1 & $1: 3$ & 2 & 3 & 2 & 2 & 3 & 2 & 2 & 1 & 2 \\
2 & $1: 3$ & 2 & 2 & 2 & 2 & 3 & 2 & 3 & 2 & 2 \\
3 & $1: 3$ & 2 & 3 & 2 & 1 & 2 & 2 & 3 & 2 & 2 \\
\hline \hline \multicolumn{2}{||c||}{$N_{i}, N_{j}$ quadratic } & \multicolumn{3}{|c||}{$I_{T, 1}^{1}$} & \multicolumn{3}{|c||}{$I_{T, 1}^{2}$} & \multicolumn{3}{|c||}{$I_{T, 1}^{3}$} \\
\hline$i$ & $j$ & $q_{11}$ & $q_{12}$ & $q_{13}$ & $q_{21}$ & $q_{22}$ & $q_{23}$ & $q_{31}$ & $q_{32}$ & $q_{33}$ \\
\hline 1 & $1: 6$ & 2 & 3 & 2 & 2 & 3 & 2 & 3 & 2 & 2 \\
2 & $1: 6$ & 2 & 2 & 2 & 2 & 3 & 2 & 3 & 2 & 2 \\
3 & $1: 6$ & 2 & 3 & 2 & 2 & 2 & 2 & 3 & 2 & 2 \\
4 & $1: 6$ & 2 & 2 & 2 & 2 & 3 & 2 & 3 & 2 & 2 \\
5 & $1: 6$ & 2 & 2 & 2 & 2 & 2 & 2 & 3 & 2 & 2 \\
6 & $1: 6$ & 2 & 3 & 2 & 2 & 2 & 2 & 3 & 2 & 2 \\
\hline
\end{tabular}

Table 10. The best choice for the exponents $q_{l m}, l, m=1,2,3$.

$$
\begin{aligned}
I_{T, 2}= & \int_{0}^{1} d x_{1} \int_{0}^{1-x_{1}} N_{i}\left(x_{1}, x_{2}\right) N_{j}\left(x_{1}, x_{2}\right) d x_{2} \\
& \int_{0}^{1} d y_{1} \int_{0}^{1-y_{1}} \frac{d y_{2}}{\left\{\left[a_{1}\left(x_{1}-y_{1}\right)+b_{1}\left(x_{2}-y_{2}\right)\right]^{2}+\left[a_{2}\left(x_{1}-y_{1}\right)+b_{2}\left(x_{2}-y_{2}\right)\right]^{2}\right\}^{3 / 2}} \\
= & \frac{1}{|A|^{2}} \int_{0}^{1} d x_{1} \int_{0}^{1-x_{1}} K_{i, j}\left(x_{1}, x_{2}\right)\left[\frac{\sqrt{a x_{1}^{2}+b\left(x_{2}-1\right)^{2}+2 c x_{1}\left(x_{2}-1\right)}}{x_{1}\left(x_{1}+x_{2}-1\right)}\right. \\
& \left.-\frac{\sqrt{a x_{1}^{2}+b x_{2}^{2}+2 c x_{1} x_{2}}}{x_{1} x_{2}}+\frac{\sqrt{a\left(x_{1}-1\right)^{2}+b x_{2}^{2}+2 c x_{2}\left(x_{1}-1\right)}}{x_{2}\left(x_{1}+x_{2}-1\right)}\right] d x_{2} \\
= & : \frac{1}{|A|^{2}}\left[I_{T, 2}^{1}+I_{T, 2}^{2}+I_{T, 2}^{3}\right],
\end{aligned}
$$

where

$$
\begin{aligned}
K_{i, j}\left(x_{1}, x_{2}\right) & =N_{i}\left(x_{1}, x_{2}\right) N_{j}\left(x_{1}, x_{2}\right), \\
a=a_{1}^{2}+a_{2}^{2}, \quad b & =b_{1}^{2}+b_{2}^{2}, \quad c=a_{1} b_{1}+a_{2} b_{2} .
\end{aligned}
$$

To compute $I_{T, 2}^{1}$ we proceed as follows:

$$
\begin{array}{rl}
I_{T, 2}^{1}=\int_{0}^{1} & d x_{1} \int_{0}^{1-x_{1}}\left[K_{i, j}\left(x_{1}, x_{2}\right) \sqrt{a x_{1}^{2}+b\left(x_{2}-1\right)^{2}+2 c x_{1}\left(x_{2}-1\right)}\right. \\
& -\sqrt{b}\left(1-x_{1}-x_{2}\right) K_{i, j}\left(0, x_{2}\right) \\
& \left.-\sqrt{a+b-2 c} x_{1} K_{i, j}\left(x_{1}, 1-x_{1}\right)\right] /\left[x_{1}\left(x_{1}+x_{2}-1\right)\right] d x_{2}
\end{array}
$$




$$
\begin{aligned}
& -\sqrt{b} \int_{0}^{1} \frac{d x_{1}}{x_{1}} \int_{0}^{1-x_{1}} K_{i, j}\left(0, x_{2}\right) d x_{2} \\
& +\sqrt{a+b-2 c} \int_{0}^{1} K_{i, j}\left(x_{1}, 1-x_{1}\right) d x_{1} \int_{0}^{1-x_{1}} \frac{d x_{2}}{x_{1}+x_{2}-1} \\
= & : I_{T, 2}^{1,1}+I_{T, 2}^{1,2}+I_{T, 2}^{1,3}
\end{aligned}
$$

As regards $I_{T, 2}^{1,1}$, first we introduce the changes of variable $x_{1}=\eta$ and $x_{2}=$ $-\xi-\eta+1$, with $0 \leq \xi \leq 1$ and $0 \leq \eta \leq 1-\xi$, and then we apply the Duffy transformation $\xi=u v$ and $\eta=(1-u) v$, with $0 \leq u, v \leq 1$; hence, we have

$$
\begin{aligned}
I_{T, 2}^{1,1}= & -\int_{0}^{1} d \xi \int_{0}^{1-\xi}\left[K_{i, j}(\eta,-\xi-\eta+1) \sqrt{a \eta^{2}+b(\xi+\eta)^{2}-2 c \eta(\xi+\eta)}\right. \\
& \left.-\sqrt{b} \xi K_{i, j}(0,-\xi-\eta+1)-\sqrt{a+b-2 c} \eta K_{i, j}(\eta, 1-\eta)\right] /(\xi \eta) d \eta \\
= & -\int_{0}^{1} d u \int_{0}^{1}\left[K_{i, j}((1-u) v, 1-v) \sqrt{a(1-u)^{2}+b-2 c(1-u)}\right. \\
& -\sqrt{b} u K_{i, j}(0,1-v) \\
& \left.-\sqrt{a+b-2 c}(1-u) K_{i, j}((1-u) v, 1-(1-u) v)\right] /[u(1-u)] d v .
\end{aligned}
$$

Moreover,

$$
I_{T, 2}^{1,2}=-\sqrt{b} \int_{0}^{1} \frac{\left[\int_{0}^{1-x_{1}} K_{i, j}\left(0, x_{2}\right) d x_{2}-\int_{0}^{1} K_{i, j}\left(0, x_{2}\right) d x_{2}\right]}{x_{1}} d x_{1}
$$

and

$$
I_{T, 2}^{1,3}=-\sqrt{a+b-2 c} \int_{0}^{1} K_{i, j}\left(x_{1}, 1-x_{1}\right) \log \left(1-x_{1}\right) d x_{1} .
$$

To compute $I_{T, 2}^{2}$, we first rewrite it as follows:

$$
\begin{aligned}
I_{T, 2}^{2}= & -\int_{0}^{1} d x_{1} \int_{0}^{1-x_{1}}\left[K_{i, j}\left(x_{1}, x_{2}\right) \sqrt{a x_{1}^{2}+b x_{2}^{2}+2 c x_{1} x_{2}}\right. \\
& \left.-\sqrt{a} x_{1} K_{i, j}\left(x_{1}, 0\right)-\sqrt{b} x_{2} K_{i, j}\left(0, x_{2}\right)\right] /\left(x_{1} x_{2}\right) d x_{2} \\
& -\sqrt{a} \int_{0}^{1} K_{i, j}\left(x_{1}, 0\right) \log \left(1-x_{1}\right) d x_{1} \\
& -\sqrt{b} \int_{0}^{1} \frac{\left[\int_{0}^{1-x_{1}} K_{i, j}\left(0, x_{2}\right) d x_{2}-\int_{0}^{1} K_{i, j}\left(0, x_{2}\right) d x_{2}\right]}{x_{1}} d x_{1} \\
=: & I_{T, 2}^{2,1}+I_{T, 2}^{2,2}+I_{T, 2}^{1,2},
\end{aligned}
$$


being the latter integral equal to that defined in (3.19). We then introduce in $I_{T, 2}^{2,1}$ the Duffy transformation $x_{1}=u v$ and $x_{2}=(1-u) v, 0 \leq u, v \leq 1$; we obtain

$$
\begin{aligned}
I_{T, 2}^{2,1}= & -\int_{0}^{1} d u \int_{0}^{1}\left[K_{i, j}(u v,(1-u) v) \sqrt{a u^{2}+b(1-u)^{2}+2 c u(1-u)}\right. \\
& \left.-\sqrt{a} u K_{i, j}(u v, 0)-\sqrt{b}(1-u) K_{i, j}(0,(1-u) v)\right] /[u(1-u)] d v .
\end{aligned}
$$

Finally, we treat $I_{T, 2}^{3}$ in an analogous way to $I_{T, 2}^{1}$; therefore, first we write

$$
\begin{aligned}
I_{T, 2}^{3}= & \int_{0}^{1} d x_{1} \int_{0}^{1-x_{1}}\left[K_{i, j}\left(x_{1}, x_{2}\right) \sqrt{a\left(x_{1}-1\right)^{2}+b x_{2}^{2}+2 c x_{2}\left(x_{1}-1\right)}\right. \\
& -\sqrt{a}\left(1-x_{1}-x_{2}\right) K_{i, j}\left(x_{1}, 0\right) \\
& \left.-\sqrt{a+b-2 c} x_{2} K_{i, j}\left(x_{1}, 1-x_{1}\right)\right] /\left[x_{2}\left(x_{1}+x_{2}-1\right)\right] d x_{2} \\
& -\sqrt{a} \int_{0}^{1} K_{i, j}\left(x_{1}, 0\right) \log \left(1-x_{1}\right) d x_{1} \\
& -\sqrt{a+b-2 c} \int_{0}^{1} K_{i, j}\left(x_{1}, 1-x_{1}\right) \log \left(1-x_{1}\right) d x_{1} \\
= & I_{T, 2}^{3,1}+I_{T, 2}^{2,2}+I_{T, 2}^{1,3},
\end{aligned}
$$

being the second and the third integral at the right-hand side of (3.23) equal to $I_{T, 2}^{2,2}$ and $I_{T, 2}^{1,3}$ defined in (3.21) and (3.20), respectively. Hence, we rewrite $I_{T, 2}^{3,1}$ by introducing first the changes of variable $x_{1}=-\xi-\eta+1$ and $x_{2}=\eta$, with $0 \leq \xi \leq 1$ and $0 \leq \eta \leq 1-\xi$, and then the Duffy transformation $\xi=u v$ and $\eta=(1-u) v$, with $0 \leq u, v \leq 1$ :

$$
\begin{aligned}
I_{T, 2}^{3,1}= & -\int_{0}^{1} d \xi \int_{0}^{1-\xi}\left[K_{i, j}(-\xi-\eta+1, \eta,) \sqrt{a(\xi+\eta)^{2}+b \eta^{2}-2 c \eta(\xi+\eta)}\right. \\
& \left.-\sqrt{b} \xi K_{i, j}(-\xi-\eta+1,0)-\sqrt{a+b-2 c} \eta K_{i, j}(-\xi-\eta+1, \xi+\eta)\right] /(\xi \eta) d \eta \\
= & -\int_{0}^{1} d u \int_{0}^{1}\left[K_{i, j}(1-v,(1-u) v) \sqrt{a+b(1-u)^{2}-2 c(1-u)}\right. \\
& \left.-\sqrt{a} u K_{i, j}(1-v, 0)-\sqrt{a+b-2 c}(1-u) K_{i, j}(1-v, v)\right] /[u(1-u)] d v .
\end{aligned}
$$


By taking into account equations (3.17)-(3.24), from (3.16) we derive that

$$
I_{T, 2}=\frac{1}{|A|^{2}}\left[I_{T, 2}^{1,1}+I_{T, 2}^{2,1}+I_{T, 2}^{3,1}+2\left(I_{T, 2}^{1,2}+I_{T, 2}^{2,2}+I_{T, 2}^{1,3}\right)\right] .
$$

The first three integrals are evaluated by using the $n$-point Gauss-Legendre formula, while the remaining ones are computed by using the same Gaussian rule, after having introduced our smoothing change of variable (2.4) with $q_{1}=3$.

In Table 11 we have reported the relative errors for $I_{T_{a}}$ obtained in the case of the triangle $T_{a}$ with corners at $(0,0),(a, 1),(0,1)$, with $a$ varying in $\{1,1 / 2,1 / 4\}$ and by using linear functions $N_{i}, N_{j}$.

\begin{tabular}{||c|c|c||c||c||c||}
\hline$i$ & $j$ & $n$ & $T_{1}$ & $T_{1 / 2}$ & $T_{1 / 4}$ \\
\hline 1 & 1 & 6 & $7.28-05$ & $7.09-05$ & $3.15-04$ \\
& & 8 & $1.36-05$ & $8.87-06$ & $2.84-05$ \\
& & 10 & $4.00-06$ & $1.95-06$ & $5.67-06$ \\
\hline 1 & 2 & 6 & $7.59-04$ & $3.04-03$ & $1.83-03$ \\
& & 8 & $3.53-05$ & $1.86-04$ & $1.51-04$ \\
\hline 1 & 3 & 6 & $3.15-04$ & $2.90-04$ & $1.73-05$ \\
& & 8 & $5.38-06$ & $3.08-05$ & $3.30-05$ \\
\hline 2 & 1 & 6 & $2.89-03$ & $1.87-02$ & $3.42-03$ \\
& & 8 & $3.32-04$ & $1.52-03$ & $1.89-04$ \\
\hline 2 & 2 & 6 & $3.42-04$ & $1.66-04$ & $4.48-04$ \\
& & 8 & $6.29-05$ & $7.75-06$ & $4.65-05$ \\
& & 10 & $2.08-05$ & $2.36-05$ & $2.55-05$ \\
\hline 2 & 3 & 6 & $1.22-03$ & $4.75-04$ & $9.06-05$ \\
& & 8 & $4.27-04$ & $7.34-05$ & $9.46-05$ \\
& & 10 & $9.91-05$ & $2.08-05$ & $6.64-06$ \\
\hline 3 & 1 & 6 & $4.05-04$ & $2.20-04$ & $3.91-04$ \\
& & 8 & $2.11-05$ & $7.94-06$ & $1.04-06$ \\
& & 10 & $1.01-05$ & $2.85-06$ & $4.38-06$ \\
\hline 3 & 2 & 6 & $3.05-04$ & $2.22-04$ & $7.07-04$ \\
& & 8 & $7.15-06$ & $2.01-05$ & $7.97-05$ \\
& & 10 & $2.93-05$ & $3.97-06$ & $3.17-06$ \\
\hline 3 & 3 & 6 & $3.47-05$ & $3.85-06$ & $3.14-05$ \\
& & 8 & $3.25-06$ & $2.88-06$ & $8.62-06$ \\
& & 10 & $5.01-07$ & $5.50-08$ & $8.39-07$ \\
\hline
\end{tabular}

Table 11. Relative errors for $I_{T_{a}}$, where $T_{a}$ has corners at $(0,0),(\mathrm{a}, 1),(0,1)$ and with $N_{i}, N_{j}$ linear.

In Table 12 we have reported the relative errors obtained for $I_{T_{a}}$, on the same triangle $T_{a}$ of Table 11 and by using quadratic functions $N_{i}, N_{j}$.

Remark. We explicitly remark that if we apply the above numerical procedure without introducing smoothing transformations, we generally obtain orders of relative accuracy halved with respect to those reported in the Tables 11 and 12. Moreover, notice that for simplicity we have considered in Table 10 only a choice for the smoothing exponents with varying $\left\{N_{j}\right\}, j=1, \ldots, 6$. 


\begin{tabular}{||c|c|c||c|c||c|c||c|c||}
\hline$i$ & $j$ & $n$ & \multicolumn{2}{|c||}{$T_{1}$} & \multicolumn{2}{c||}{$T_{1 / 2}$} & \multicolumn{2}{c||}{$T_{1 / 4}$} \\
\hline & & & $\min$ & $\max$ & $\min$ & $\max$ & $\min$ & $\max$ \\
\hline 1 & $1: 6$ & 6 & $5.10-05$ & $3.54-03$ & $7.79-04$ & $2.89-02$ & $9.69-05$ & $2.22-01$ \\
& & 8 & $1.29-05$ & $6.68-05$ & $2.42-05$ & $8.00-04$ & $1.60-05$ & $7.54-02$ \\
& & 10 & $3.18-06$ & $4.71-05$ & $1.24-06$ & $4.65-05$ & $3.85-06$ & $1.72-03$ \\
\hline 2 & $1: 6$ & 6 & $4.88-04$ & $4.28-03$ & $1.27-04$ & $9.69-03$ & $1.21-04$ & $1.24-01$ \\
& & 8 & $1.21-05$ & $5.86-04$ & $4.33-07$ & $5.48-04$ & $5.20-05$ & $3.40-03$ \\
& & 10 & $1.73-05$ & $1.58-04$ & $1.30-05$ & $5.29-04$ & $3.98-06$ & $9.05-03$ \\
\hline 3 & $1: 6$ & 6 & $3.47-05$ & $4.29-03$ & $8.61-05$ & $1.31-03$ & $8.29-05$ & $3.73-03$ \\
& & 8 & $2.12-05$ & $1.03-04$ & $4.09-06$ & $7.28-05$ & $9.73-06$ & $9.87-05$ \\
& & 10 & $9.15-07$ & $2.73-05$ & $3.01-07$ & $7.62-06$ & $7.14-07$ & $7.69-05$ \\
\hline 4 & $1: 6$ & 6 & $5.42-04$ & $2.65-03$ & $1.45-05$ & $9.79-03$ & $7.73-05$ & $4.96-03$ \\
& & 8 & $7.80-06$ & $4.60-05$ & $1.02-05$ & $1.46-04$ & $8.53-06$ & $4.09-04$ \\
& & 10 & $2.36-06$ & $3.11-05$ & $2.56-06$ & $5.07-05$ & $4.62-06$ & $8.21-05$ \\
\hline 5 & $1: 6$ & 6 & $4.78-05$ & $3.49-03$ & $3.15-05$ & $4.44-04$ & $1.06-04$ & $2.25-03$ \\
& & 8 & $2.37-06$ & $7.88-05$ & $5.59-06$ & $8.98-05$ & $3.39-05$ & $6.84-05$ \\
& & 10 & $1.58-06$ & $2.83-05$ & $1.08-07$ & $1.08-05$ & $4.21-06$ & $2.16-05$ \\
\hline 6 & $1: 6$ & 6 & $8.77-05$ & $4.76-03$ & $2.64-04$ & $1.23-03$ & $5.72-05$ & $2.01-03$ \\
& & 8 & $4.22-06$ & $6.83-05$ & $4.00-06$ & $1.06-04$ & $5.85-05$ & $1.38-04$ \\
& & 10 & $2.14-06$ & $1.18-05$ & $1.54-06$ & $1.65-05$ & $2.11-06$ & $1.57-05$ \\
\hline
\end{tabular}

Table 12. Minimum and maximum relative errors for $I_{T_{a}}$, where $T_{a}$ has corners at $(0,0),(\mathrm{a}, 1),(0,1)$ and with $N_{i}, N_{j}$ quadratic.

Obviously this fact has involved in some particular cases a loss of precision; indeed, in these cases a suitable choice would have given a more accurate relative precision than that of the Tables 11 and 12 .

\section{References}

[1] Andrä, H. and Schnack, E., Integration of singular Galerkin-type boundary element integrals for 3D elasticity problems, Numer. Math., 76 (1997), 143-165.

[2] Diligenti, M. and Monegato, G., Integral evaluation in the BEM solution of (hyper)singular integral equations. 2D problems on polygonal domains, J. Comput. Appl. Math., 81 (1997), 29-57.

[3] Elliott, D. and Prössdorf, S., An algorithm for the approximate solution of integral equations of Mellin type, Numer. Math., 70 (1995), 427-452.

[4] Elliott, D. and Venturino, E., Sigmoidal transformations and the Euler-Maclaurin expansion for evaluating certain Hadamard finite-part integrals, Numer. Math., 77 (1997), 453-465.

[5] Haas, M. and Kuhn, G., A symmetric Galerkin BEM implementation for 3D elastostatic problems with an extension to curved elements, Comp. Mech., 28 (2002), 250-259.

[6] Korobov, N. M., Number-Theoretic Methods of Approximate Analysis, GIFL, Moscow, 1963.

[7] Monegato, G., Numerical evaluation of hypersingular integrals, J. Comput. Appl. Math., 50 (1994), 9-31.

[8] Monegato, G. and Scuderi, L., High order methods for weakly singular integral equations with non smooth input functions, Math. Comp., 67 (1998), 1493-1515.

[9] , Numerical integration of functions with boundary singularities, J. Comput. Appl. Math., Special Issue: "Numerical Evaluation of Integrals", D. Laurie, R. Cools eds., 112 (1999), 201-214. 
[10] Mori, M. and Sugihara, M., The double-exponential transformation in numerical analysis, J. Comput. Appl. Math., Numerical Analysis 2000, Vol. V, Quadrature and orthogonal polynomials, 127 (2001), 287-296.

[11] Prössdorf, S. and Rathsfeld, A., Quadrature methods for strongly elliptic Cauchy singular integral equations on an interval, in: H. Dym (Ed.), The Gohberg Anniversary Collection, Vol. 2: Topics in Analysis and Operator Theory, Birkhäuser, Basel, (1991), 435-471.

[12] Sauter, S. and Lage, C., Transformation of hypersingular integrals and black-box cubature, Math. Comp., 70 (2000), 223-250.

[13] Sauter, S. and Schwab, C., Quadrature for $h p$-Galerkin BEM in $\mathbb{R}^{3}$, Numer. Math., 78 (1997), 211-258.

[14] Sidi, A., A new variable transformation for numerical integration, in: H. Braess, G. Hämmerlin, eds., Numerical Integration IV, Birkhäuser Verlag, Basel, ISNM 112 (1993), 359-373.

[15] Sugihara, M., Optimality of the double exponential formula - functional analysis approach, Numer. Math., 75 (1997), 379-395.

[16] Takahasi, H. and Mori, M., Double exponential formulas for numerical integration, Publ. RIMS, Kyoto Univ., 9 (1974), 721-741. 